CAHIERS DE

NARRATOLOGIE
Cahiers de Narratologie

Analyse et théorie narratives

15 | 2008

Récits et genres historiques

\title{
El húsar, un exemple de fiction historique
}

\author{
Marie-Thérèse Garcia
}

\section{OpenEdition}

Journals

Édition électronique

URL : http://journals.openedition.org/narratologie/785

DOI : $10.4000 /$ narratologie.785

ISSN : 1765-307X

Éditeur

LIRCES

\section{Référence électronique}

Marie-Thérèse Garcia, "El húsar, un exemple de fiction historique », Cahiers de Narratologie [En ligne], 15 | 2008, mis en ligne le 14 décembre 2008, consulté le 15 novembre 2019. URL : http:// journals.openedition.org/narratologie/785; DOI : 10.4000/narratologie.785

Ce document a été généré automatiquement le 15 novembre 2019.

\section{(c) (i) $(9)$}

Cahiers de Narratologie - Analyse et théorie narratives est mis à disposition selon les termes de la licence Creative Commons Attribution - Pas d'Utilisation Commerciale - Pas de Modification 4.0 International. 


\title{
El húsar, un exemple de fiction historique
}

\author{
Marie-Thérèse Garcia
}

Créateur d'un territoire romanesque qui se situe à la croisée des chemins entre littérature populaire et littérature érudite, Arturo Pérez-Reverte, écrivain éminemment médiatique élu académicien en 2003, a acquis une notoriété qui dépasse largement les frontières de l'Espagne. L'auteur de $E l$ húsar ${ }^{1}$, membre assidu du «club Dumas » et sociétaire à ses heures du très fermé cercle borgésien, récupère et revisite des formules génériques empruntées à la grande tradition de la littérature populaire du $\mathrm{XIX}^{\mathrm{e}}$ siècle telles que le feuilleton historique ${ }^{2}$, le roman d'aventures ${ }^{3}$, le roman policier ${ }^{4}$. En dépit de la grande variété des genres romanesques cultivés, le lecteur de Pérez-Reverte ne manquera pas de constater que, dans la production littéraire du romancier, la thématique historique joue toujours un rôle, occupant une place centrale ou périphérique selon la pratique d'écriture mise en œuvre. L'un de ses plus grands succès de librairie, Las aventuras del Capitán Alatriste, une série qui compte aujourd'hui déjà six volumes, est consacré à la récupération d'une mémoire considérée comme fondatrice par l'auteur : le Siècle d'Or espagnol avec ses ombres et ses lumières. En outre, deux des trois derniers ouvrages récemment publiés confirment l'engouement d'Arturo PérezReverte pour la narration de l'Histoire : Cabo Trafalgar ${ }^{5}$ et Un día de cólera ${ }^{6}$ proposent deux récits réalistes à peine romancés et documentés avec rigueur sur le désastre naval de Trafalgar pour le premier et sur la résistance héroïque du peuple madrilène les 2 et 3 mai 1808 pour le second. Il n'est donc pas surprenant que Pérez-Reverte ait choisi aussi le champ de l'Histoire pour son entrée en littérature en 1986 avec El húsar. Entre nouvelle, «novela corta » et roman, peu remarquée lors de sa publication, cette œuvre semble maintenant reconnue par la critique comme une ópera prima prometteuse, portant en elle les empreintes génétiques de l'écriture revertienne. Le monde éditorial lui accorde aujourd'hui une seconde chance: Alfaguara republie cet ouvrage en Espagne et en France les éditions du Seuil ont édité en avril 2005 la traduction de François Maspéro ${ }^{7}$. Ce court récit ne prétend pas au statut de fresque historique et échappe à toute classification générique. Il est, à plus d'un titre, un roman des origines : d'abord parce qu'il est le premier d'une longue lignée, fondateur de l'esthétique de 
l'écrivain. Ensuite parce qu'il est aussi « un roman familial » qui retrace la destinée poétique de Frédéric Gluntz - alias Jean Gall - soldat de la grande armée et ancêtre alsacien d'Arturo Pérez-Reverte. Enfin parce que la représentation de cette bataille fictive s'inscrit dans une période-clé de l'histoire du peuple espagnol et de l'épopée napoléonienne : la guerre de l'Indépendance. Un moment privilégié où s'exprime en Espagne la conscience de l'idée de patrie, une époque où se manifestent les frémissements d'un sentiment d'identité nationale. Bien que le classement d'une œuvre de Pérez-Reverte dans une taxinomie soit toujours tâche délicate puisque le romancier de Carthagène pratique à plaisir, comme beaucoup de ses contemporains, l'hybridation des genres, El húsar me semble un exemple de fiction historique dans la mesure où la recréation poétique du passé est investie d'une fonction diégétique et est sentie comme préhistoire concrète du présent selon l'expression de Georg Lukács. En perpétuelle tension entre historicisation de la fiction et fictionalisation de l'Histoire, la narration du voyage au bout de la nuit du hussard Frédéric Glüntz s'impose au fil du texte comme une page d'intrahistoire vraie et historiquement justifiée. Le contexte historique, qui pourrait apparaître comme un simple décor " prétextuel " pour une dénonciation de la guerre, assume dans ce récit au moins trois fonctions: il structure la narration, engendre des créatures, héros moyens animés d'une conscience historique de leur existence, et les inscrit dans un passé ressuscité par des procédés rhétoriques de contextualisation, générateurs d'un effet de réel historique. Le premier volet de mon étude portera donc sur la fonction diégétique du contexte historique et sur la charge symbolique dont il est investi. Le deuxième tentera de montrer comment le personnage de Frédéric Glüntz, " héros moyen », porteur de toute l'architecture narrative, accède au statut de créature historique. Enfin, j'étudierai certaines des voies littéraires et iconographiques par lesquelles transite la contextualisation - malgré des sources historiographiques déclarées - et grâce auxquelles la refiguration poétique de cette journée de combat de la Guerre de l'Indépendance revêt des accents d'authenticité qui la haussent au niveau de témoignage vécu.

Dans El húsar, récit structuré en sept unités symboliques qui vont de «la nuit à la gloire ", Pérez-Reverte choisit pour période de référence les événements de la guerre de l'Indépendance, un moment de crise nationale déterminant pour la formation de l'Espagne des $\mathrm{XIX}^{e}$ et $\mathrm{XX}^{\mathrm{e}}$ siècles, une époque charnière pour la construction de la nation. La puissante armée napoléonienne qui vient d'envahir la péninsule ibérique y subit de lourdes pertes et se trouve engagée malgré elle dans une guérilla sans merci. Le peuple espagnol, pourtant abandonné par ses souverains Charles IV, Marie-Louise et Ferdinand VII qui, obéissant à Napoléon, se sont rendus à Bayonne et ont abdiqué, prend fait et cause pour les Bourbons et se mutine contre les Français. Les forces populaires découvrent le pouvoir qu'elles représentent et prennent en charge l'honneur et la défense du pays. La guerre de l'Indépendance, affrontement entre l'armée de Joseph $1^{\text {er }}$ contre les troupes espagnoles et celles de leurs alliés angloportugais est aussi un soulèvement national. Cette duplicité en fait un conflit où la ruse l'emporte sur le déploiement de puissance, et Napoléon dans Le Mémorial de SainteHélène reconnaîtra avoir été vaincu par un peuple plus que par une armée. Le narrateur de $E l$ húsar montre à maintes reprises le désarroi des hussards confrontés à une guérilla dont les règles ne figurent dans aucun manuel militaire. Dans une « note de l'auteur $»^{8}$, 
placée en fin de narration, Pérez-Reverte revendique la nature fictionnelle de ce premier récit :

Peut-être que les spécialistes pointilleux découvriront certaines inexactitudes dans l'histoire que je viens de rapporter. Ça ne serait pas étonnant, étant donné qu'en 1808 en Andalousie, on n'a livré aucune bataille ressemblant à celle décrite dans le roman, à l'exception de Bailén qui a eu lieu dans des circonstances bien différentes. D'autre part l'attribution des noms des unités françaises qui interviennent dans la narration a été dictée par le hasard, pour des raisons évidentes dans une trame de fiction comme celle qui nous occupe. Il y a cependant une exception: le 4e Régiment de Hussards a été effectivement détaché en Espagne entre 1808 et 1813 et a pris part à des actions comme Belchite, Alcañiz, Estela, Chiclana, Sagunto, Yecla y Paso de Ordás. J'ignore si son premier escadron fut anéanti au cours de l'un de ces combats, mais j'ai de sérieuses raisons d'en douter car je n'ai trouvé dans aucun des nombreux documents consultés une preuve de cette disparition... Cependant, la fiction confère parfois à l'auteur l'amusant privilège de jouer des tours à l'histoire 9 .

Toujours dans la même note, et dans les lignes qui suivent, le romancier insiste sur le travail méticuleux de documentation effectué et cite des sources bibliographiques manuels d'historiens, dictionnaires et documents iconographiques - qui ont présidé à la genèse du texte et qui prétendent asseoir le roman sur des bases historiques dignes de foi. Dans ce péritexte auctorial qui fonctionne comme un seuil brutal de retour au réel, le romancier souligne donc l'écart qui s'instaure dans le récit entre Histoire et fiction tout en affirmant aussi sa volonté de respecter un contrat de "véridicité". Contrat de véridicité, certes, mais non de vérité. En effet, les faits relatés sont indéniablement fictionnels : le jeune Frédéric Gluntz, fraîchement émoulu de son école militaire, a été affecté à l'été 1808 au IV régiment de hussards, détaché en Espagne. En revanche, sa trajectoire de vie s'inscrit dans un passé attesté par l'historiographie et répond à une logique historique : la première bataille du jeune Gluntz se serait déroulée en Andalousie tout près de Bailén et peu de temps après cette défaite française dont l'horrible souvenir hante la mémoire des protagonistes. Le mensonge fictionnel n'entache pas la vraisemblance du récit et ne change rien à la signifiance historique du texte. Qu'importe le lieu référentiel fictionnel, cette histoire pourrait bien être celle de la bataille de Bailén ${ }^{10}$ vue dans la perspective des occupants. En ce sens, Arturo PérezReverte semble suivre ici le précepte énoncé par Aristote dans La Poétique selon lequel «[...] ce n'est pas de raconter les choses réellement arrivées qui est l'œuvre du poète mais bien de raconter ce qui pourrait arriver. Les événements sont possibles suivant la vraisemblance ou la nécessité. $\aleph^{11}$ Le monde fictionnel représenté dans $E l$ húsar se distingue peu de ce que les philosophes nomment un " univers possible " ${ }^{12}$, et l'auteur y représente un univers possible par sa double condition de vraisemblance et de nécessité. Parce qu'elle donne sa vraisemblance et sa nécessité à la fable, l'Histoire y est omniprésente et sous-tend toute la narration. Le narrateur consacre par exemple une longue analepse au rappel des circonstances de l'invasion de l'Espagne et au non-sens historique commis par Napoléon. L'ombre de l'Aigle et son manque de lucidité politique, responsable de cette tragédie, planent sur tout le récit. Car c'est l'entêtement bien réel de l'Empereur qui est la condition nécessaire à l'existence fictionnelle de Frédéric et de ses compagnons d'armes. Juniac, Michel de Bourmont, Frédéric, créatures poétiques, périront parce que le vainqueur d'Austerlitz, figure historique, n'a pas pris la juste mesure de cet ennemi si différent des autres comme le soutient avec conviction don Álvaro del Vigal, l'aristocrate « afrancesado » rencontré par Frédéric :

Il regrettait néanmoins que Napoléon n'ait pas encore compris que l'Espagne ne pouvait être mesurée à l'aune du reste des autres pays européens. [...] 
L'Espagne est une nation très ancienne, orgueilleuse et fidèle à ses mythes, que ceux-ci soient justifiés ou non. Bonaparte est tellement habitué à voir les peuples s'agenouiller, qu'il ne peut concevoir, ce qui est une grave erreur d'appréciation, qu'il y a de ce côté des Pyrénées une race décidée à ne pas accepter sa volonté. ${ }^{13}$

L'Histoire, condition nécessaire à la construction de l'univers fictionnel de El húsar, structure la fiction. Grâce à la guerre de l'Indépendance, quatre éléments constitutifs fondamentaux de la construction narrative se trouvent justifiés : le temps et l'espace référentiels, les personnages principaux et secondaires et l'action. Dès les premières pages, le dialogue entre Frédéric Glüntz et Michel de Bourmont, témoin et acteur des combats madrilènes des 2 et 3 mai 1808, présente le cadre spatio-temporel : une drôle de guerre qui oppose "des bandes de patriotes guérilleros" à l'armée la mieux entraînée d'Europe. Une guerre qui est moins « une guerre d'ensemble qu'une série de guerres locales, une guerre qui n'est pas une lutte de souverain mais la révolte d'un peuple $~^{14}$ selon les termes de Georges Roux. Cette campagne « où l'on voyait à peine le visage de l'ennemi » défie toutes les leçons de stratégie dont le brillant élève de l'école militaire pensait se prévaloir; ce n'est pas la guerre dont rêvaient Frédéric et ses frères d'armes. L'ennemi est partout mais on ne le voit nulle part. «[...] ici même les chiens, les oiseaux, le soleil et les pierres sont nos ennemis $»^{15}$ dira de Bourmont, comme si la terre même d'Espagne devenait acteur de cette résistance et refusait de se voir occupée. Ici, point de place pour les actions d'éclat, il s'agit de savoir déjouer les embuscades, de demeurer en permanence aux aguets, de se méfier de tout, même de l'eau des puits. Ici la bataille perd toute théâtralité de parade pour devenir une lutte primitive au corps à corps.

5 Le contexte historique situe, structure l'action et assume aussi une fonction prophétique. Il est annonciateur du destin funeste qui attend nos jeunes recrues après une véritable via crucis. Et en effet, tout au long du texte, l'évocation de Bailén ou des journées des 2 et 3 mai reviendra en leitmotiv, tel un chœur antique, dans des analepses dont la fonction n'est pas seulement commémorative mais prémonitoire. Cependant personne ne veut entendre le présage ; tous, Dombrowski, Letac et bien sûr Napoléon, écartent dédaigneusement ce qui ne peut être qu'un revers occasionnel :

Pourtant tous étaient convaincus, et Frédéric le premier qu'une telle situation ne pourrait se prolonger longtemps. La dureté et la profusion des châtiments exemplaires ne tarderaient pas à faire rentrer le torrent dans son lit. Il suffisait de pendre et de fusiller davantage de cette racaille inculte et fanatique, achevant ainsi une fois pour toutes la pacification de l'Espagne, pour pouvoir continuer à se consacrer à de plus glorieuses entreprises ${ }^{16}$.

Pacifier, civiliser, apporter les lumières à une masse ignorante, telles sont les missions supérieures dont se sentent investis les personnages. Le jugement émis par de Bourmont sur l'ennemi qu'ils combattent, l'Espagnol, c'est-à-dire le pays entier comme le dit Frédéric, est empreint d'un dédain, perceptible dans le choix de termes tels que "populace informe» ou " tourbe fanatique », un mépris qui rejoint celui qu'exprime l'Empereur lorsqu'il définit l'Espagne comme "une canaille de paysans conduits par une canaille de prêtres». À l'évidence ni Frédéric ni de Bourmont ne peuvent comprendre le patriotisme primitif viscéral qui se manifeste contre l'envahisseur. Cette incompréhension, cette méconnaissance de l'Espagne fut la grande erreur de Napoléon qui l'entraîna dans le guêpier espagnol. Le sentiment d'embourbement ressenti par l'Empereur tout au long de cette campagne se reflète de façon symbolique dans le récit de Pérez-Reverte. Ainsi, la bataille se déroulera sous la pluie et nos sémillants hussards qui rêvaient de l'appel glorieux des Walkyries mourront dans un bourbier obscur. À 
l'instar de Napoléon, les deux hussards ne peuvent envisager leur défaite. Durant la nuit de veille qui précède la bataille initiatique, les deux amis prennent conscience de la menace sans pourtant comprendre que cette campagne signifiera leur anéantissement; la discussion des deux officiers sur les mérites du sabre et sur les devoirs du hussard, l'évocation du duel entre de Bourmont et Fucken sont en total décalage avec la situation que les personnages vont affronter. L'idéalisme de de Bourmont et de Gluntz ne résistera pas à l'épreuve du réel. L'information historique délivrée par les dialogues ou des monologues intérieurs se charge tout au long de la narration d'un poids dramatique parce qu'elle est transmise au lecteur par des personnages qui font à leur dépens l'expérience de l'Histoire. Les diverses procédures narratives mises en œuvre ancrent le récit dans un contexte historique qui détermine le destin $\mathrm{du} \mathrm{IV}^{\mathrm{e}}$ régiment de hussards, et le passé diégétisé acquiert aussi valeur de symbole : celui de la résistance d'un peuple. Trop inadaptés pour survivre dans un univers aussi hostile, Frédéric, Michel et ses compagnons d'armes seront les acteurs d'une chronique d'une mort annoncée.

7 Comme tout auteur de roman historique, Pérez-Reverte élabore un système de personnages dans lequel les figures de l'Histoire côtoient des créations poétiques purement fictives mais tout aussi vraisemblables que les actants ayant un référent réel. Frédéric Gluntz est un de ces êtres qui fait l'expérience de l'Histoire, un de ces destins comme le dit Georg Lukacs « dans lesquels les contenus socio-humains importants, les problèmes, les courants, etc, d'une époque apparaissent». Sous-lieutenant des hussards, envoyé en Espagne pour y recevoir «son baptême de feu et de sang ", Frédéric est, comme le souligne l'article défini du titre, El húsar, l'archétype des jeunes recrues qui composaient l'essentiel de ce corps d'élite. L'Histoire lui confère son identité fictionnelle comme l'atteste cette profession de foi exaltée de Frédéric où se reflète l'esprit collectif qui l'anime :

Je crois... je crois qu'un homme comme toi, ou comme moi, ne se justifie, ne trouve sa raison d'être, que s'il chevauche le pistolet dans une main et le sabre dans l'autre, en criant « Vive l'Empereur $»^{17}$

8 Il est donc logique que le destin individuel du jeune homme intéresse à peine l'auteur qui ne consacre que quelques pages à la vie privée de Frédéric. Le propos du romancier est tout autre, Pérez-Reverte cherche à donner une description existentielle d'un "être-“dans" le temps » selon l'expression de Paul Ricoeur. Il s'agit pour l'auteur de représenter la destinée collective de jeunes soldats de la Grande armée à travers un personnage attachant. Frédéric, prédestiné par son prénom à être le héros d'une Education sentimentale, fera comme tous ses camarades un apprentissage mortel et comprendra que sa propre existence est « historiquement conditionnée ». Il verra le brillant avenir qu'il croyait s'être choisi se résoudre en une mort obscure dans la fange. La narration se concentre sur un personnage parfaitement situé dans un cadre historique, et sa trajectoire humaine et diégétique est dès les premières lignes du roman conditionnée par cette page d'Histoire. Le romancier s'attache à donner à ses personnages une particularité qui dérive de la spécificité historique de leur temps. Aussi est-il aisé de reconnaître dans Frédéric Gluntz, fils d'un riche commerçant strasbourgeois, un type humain représentatif de la petite bourgeoisie provinciale à l'époque napoléonienne. Celui-ci incarne à la fois le rêve de promotion sociale et le 
complexe d'infériorité d'une classe ascendante par rapport à une aristocratie finissante que représente Michel de Bourmont. Il entre à l'académie militaire dans l'espoir de s'élever dans l'échelle sociale et voudrait mériter par des actions héroïques un grade prestigieux à l'image du colonel Letac qui commande son régiment. Il sait cependant que même un général de l'Empire n'aura jamais cette distinction que la noblesse dominante a acquise depuis des siècles ${ }^{18}$. Les réformes engendrées par la Révolution de 1789 ont mis en place un nouvel ordre social et les nombreuses campagnes militaires menées par l'Empereur ont permis à nombre de jeunes officiers, issus de la bourgeoisie et du peuple, d'accéder à une certaine reconnaissance sociale. Napoléon ou Murat, fils d'aubergiste, ne donnent-ils pas un exemple d'irrésistible ascension? Dans El húsar, cette volonté de dépassement teintée d'une certaine frustration constitue l'un des « (...) mobiles sociaux et humains qui ont conduit ces hommes à penser et à agir précisément comme ils l'ont fait dans la réalité historique $»^{19}$. De surcroît, Frédéric est porteur des idéaux d'héroïsme et de gloire de ses compagnons d'armes :

[Dans la carrière des armes il cherchait] la cristallisation d'une aspiration désir supérieure, d'un ideal qui l'arrachait au confort de la vie bourgeoise et lui montrait le chemin de l'héroisme, des nobles sentiments, du sacrifice suprême. Il était entré dans l'armée comme on entre en religion, empoignant son sabre comme on empoigne la croix. Et si les prêtres et les pasteurs aspiraient à gagner le Ciel, il aspirait à gagner la gloire : l'admiration de ses camarades, le respect de ses chefs, sa propre estime, avec cette belle conviction désintéressée qu'il était doux et grand de combattre, de souffrir et peut-être de mourir pour une idée. L'Idée. ${ }^{20}$

$9 \quad$ L'idéalisme exalté que tous ont acquis sur les bancs de l'école militaire est la force qui les meut en tant que personnages même si elle ne résiste pas à la confrontation avec le réel. La désillusion, quand ce n'est pas une mort absurde, est au bout de leur quête de perfection qui s'apparente plutôt à un chemin de croix. Notre romantique héros ne découvrira qu'au seuil de sa mort l'inutilité de son sacrifice:

\begin{abstract}
Les vieux soldats, il le découvrait maintenant, avaient toujours raison. C'était pour ça qu'ils se taisaient. Ils savaient, et la connaissance, la sagesse les rendaient silencieux. Ils savaient, et au diable tout le reste. Mais ils ne le racontaient à personne; les vieux renards étaient malins. Oui, ils savaient que tout homme est seul au milieu des autres, et que c'est à chacun d'apprendre pour son compte. Chez eux il n'y avait pas de bravoure, il y avait de l'indifférence. Ils étaient de l'autre côté $\mathrm{du}$ mur, au-delà du bien et du mal, comme le grand-père de Frédéric, le vieux Glüntz qui s'était laissé mourir, fatigué d'attendre la mort. Il n'y avait rien à faire, le chemin était effroyablement clair. Honneur, Gloire, Patrie, Amour... Il existait un point de non-retour auquel on arrivait tôt ou tard, où tout devenait superflu, acquérait ses limites précises, sa dimension exacte. ${ }^{21}$
\end{abstract}

Le cheminement de Frédéric vers la tragique connaissance des réalités sera le même que celui de ses camarades de promotion: Philippo, Bourmont suivent le même itinéraire fatal et comme eux, il se sent à contre emploi dans cette campagne d'Espagne :

Je suis inquiet à l'idée d'être venu jusqu'ici pour tomber obscurément et sans gloire, assassiné sur un chemin solitaire par la canaille paysanne, comme le pauvre Juniac, au lieu d'être tué en chevauchant derrière l'aigle du régiment, à ciel ouvert et entouré de mes camarades, d'un coup de sabre ou d'une balle dans la poitrine, debout, bien campé sur mes étriers, l'arme à la main et la bouche pleine de sang, comme meurent les hommes. ${ }^{22}$

11 Car pour Frédéric Glüntz et pour tous les autres jeunes hussards, cette guérilla est une guerre sale, sans "brillantes charges de cavalerie», sans "mouvement de grandes 
unités ", sans " batailles aux noms glorieux qui figureraient dans les livres d'histoire et vaudraient à Frédéric Glüntz de gravir les degrés de l'honneur et de la renommée "23. Par sa soif de gloire, son idéalisme de classe et par la conscience de son rôle historique, Frédéric apparait comme un héros moyen qui incarne l'esprit d'une époque et la mentalité d'un groupe social.

Don Álvaro del Vigal est lui aussi un être de fiction profondément ancré dans une réalité historique. Il est, à l'instar de Fernando Moratín de Goya et d'autres esprits éclairés de la fin du XVIII ${ }^{e}$ siècle, le représentant de cette intelligentsia espagnole déchirée entre son attachement au progrès et à la raison qu'elle a puisé dans la fréquentation des philosophes français et le rejet de la violation du territoire national par l'armée de ce pays porteur des Lumières. Pour don Álvaro aussi c'est l'heure de vérité: il se voit obligé de prendre parti au moment de l'invasion des troupes impériales et il doit confronter sa vision libérale d'homme éclairé à la résistance « sauvage » des «bandes de guérilleros ». La rencontre de Glüntz avec le vieil aristocrate castillan permet un débat idéologique entre les points de vue français et espagnol et donne au jeune homme une chance de comprendre le sens de ce combat qui prendra sa vie, son identité et son humanité. Sans les explications de don Álvaro, la mort de Frédéric serait encore plus absurde. Le récit rend compte de cette déstructuration, de cette annihilation, de cette véritable descente aux enfers. Ainsi le narrateur s'emploie à montrer comment la bataille qui s'engage selon un bel ordonnancement de défilé militaire, se transforme peu à peu en chaos, reflétant de la sorte l'état d'âme d'un héros au départ plein de confiance et de superbe qui meurt blessé à mort et traqué comme une bête. Dans El húsar, Pérez-Reverte démontre également que le peuple fait directement l'expérience de l'Histoire. Selon Georges Lukács, les guerres napoléoniennes constituent

la possibilité concrète pour des hommes de comprendre leur propre existence comme quelque chose d'historiquement conditionné, de voir dans l'histoire quelque chose qui affecte profondément leurs vies quotidiennes et qui les concerne immédiatement. ${ }^{24}$

Cette prise de conscience des masses comme actrices de l'Histoire conditionne le rôle diégétique que leur attribue Pérez-Reverte. Ainsi, au fil du récit revertien, se profilent des groupes anonymes d'Espagnols qui incarnent cette attitude consciente d'acteurs de leur Histoire et c'est bien un groupe de partisans qui mettra fin à l'existence de Frédéric. Le romancier s'ingénie donc, dans cette première narration, à recréer les conditions socio-historiques qui ont pu générer ses créatures fictionnelles, ce qui, à mon sens, confère au temps passé représenté dans la fiction une signifiance historique.

Certes, Pérez-Reverte, grand admirateur de Pérez Galdós écrit une instantanée en négatif de Bailén mais le récit tire sa force évocatrice d'un témoin contemporain de l'événement, le peintre Francisco de Goya. En effet, plus que par la voie littéraire, la contextualisation transite par l'iconographie du grand maître aragonais. Je ne traiterai donc pas ici la filiation galdosienne de El húsar, je m'attacherai à montrer que la recréation de l'atmosphère renvoie à Los desastres de la guerra et que le recours à cette iconographie donne au texte des accents d'authenticité propres à un témoignage vécu.

Plus encore que les livres d'Histoire, les quatre-vingt-deux gravures composant Los desastres de la guerra sont inscrites de telle sorte dans l'imaginaire collectif qu'il semble impossible d'évoquer la guerre de l'Indépendance sans invoquer l'auteur des célèbres toiles représentant les 2 et 3 mai 1808. Il est donc logique qu'Arturo Pérez-Reverte 
puise aux sources iconographiques de Francisco de Goya pour ressusciter le climat de cette période tragique. En dépit de la différence des systèmes sémiotiques qui régissent message iconique et message linguistique, Los desastres de la guerra fonctionnent comme un hypotexte dans le processus d'écriture de $E l$ húsar. L'appropriation d'une œuvre picturale par nature si éloignée de l'écrit se trouve par ailleurs facilitée par l'association écriture-image que pratique Goya dans son œuvre gravée. Le texte revertien, bien qu'il soit écrit au passé, traduit la même immédiateté dans la représentation de l'horreur que celle ressentie par le spectateur devant Los desastres de la guerra ${ }^{25}$. Cette coïncidence de points de vue n'est pas fortuite, elle tient au statut de témoins oculaires de faits de guerre que durent assumer aussi bien le peintre que le romancier, à des époques différentes, bien sûr. Des sources biographiques attestent que Goya avait assisté aux journées d'horreur des 2 et 3 mai 1808, et que dès le lendemain de l'insurrection madrilène il commença ses gravures qui naissent comme un cri de désespoir devant la folie meurtrière des hommes ${ }^{26}$. La genèse de $E l$ húsar est également due au traumatisme ressenti par Pérez-Reverte alors qu'il couvrait la guerre d'Erythrée, l'un des conflits qui l'a le plus marqué dans son parcours de journaliste. Le romancier entreprend l'écriture de El húsar au sortir de ce cauchemar sanglant comme un exutoire par où s'épanche la douleur d'un homme confronté à une cruauté animale. Profondément choqués par la vision du massacre, l'artiste comme l'ancien reporter de guerre sont convaincus qu'aucun idéal, qu'aucun nationalisme ne peut justifier cette barbarie. Bárbaros ${ }^{27}$, c'est bien le terme que choisit Goya comme légende de l'une de ses planches et comme thème récurrent des Desastres de la guerra. Tout affrontement armé, pour légitime qu'on puisse le croire, ramène l'homme au rang de l'animal, prédateur ou traqué. Bouleversés par des scènes de carnage, le peintre et l'écrivain semblent s'être fixé le même objectif: témoigner pour dénoncer les désastres de la guerre et la dégradation de l'homme pris dans ses rouages infernaux. Telle est l'intention qui préside aux projets graphique et romanesque de Los Desastres et de El húsar. Cette réflexion sur la faiblesse d'une condition humaine prompte à plonger au plus profond des ténèbres est au cœur de l'œuvre des deux créateurs.

El sueño de la razón produce monstruos ${ }^{28}$ prévenait déjà Goya au bas de la gravure 43 des Caprices, mais son avertissement demeura vain et les chimères menaçantes qui servaient de fond à la gravure dévorent sauvagement leur proie dans Las resultas ${ }^{29}$ (ce qu'il en résulta), planche 72 de Los desastres de la guerra. Selon l'artiste, renoncer à la raison, c'est renoncer à la vie. Ainsi, ses 82 estampes donnent la mesure de la monstruosité de l'homme qui accepte de se laisser entraîner dans la guerre, le pire des monstres engendrés par l'égarement de la raison comme le dénonce la planche 81 : Fiero monstruo ${ }^{30}$ où une bête répugnante vautrée sur le sol se repait goulûment d'un monceau de cadavres. À l'évidence Arturo Pérez-Reverte partage ce sentiment. Lors de sa veillée d'armes, Frédéric, hanté par des rêves de gloire, embellit la mort et se sent prêt à recevoir le baiser de sa walkyrie. Les visions chimériques qui ont obscurci son entendement vont le conduire vers la mort. Il recevra son châtiment pour tant d'inconscience comme tous ceux qui se laissent «attraper dans la ronde macabre " ${ }^{31}$, une expression qui rappelle les peintures noires de La quinta del Sordo (la fameuse maison du sourd). L'éthique de Pérez-Reverte rejoint ainsi celle de Goya et la fable s'efface devant des images tout aussi barbares que les gravures goyesques pour montrer une cruauté sans limite. étrangères et de chemins qui ne mènent nulle part $~_{32}$ et l'épigraphe de Céline ${ }^{33}$ sont 
aussi tristement prémonitoires que le titre de la première gravure du peintre : «Tristes presentimientos de lo que ha de acontecer $»^{34}$. La guerre n'offre que le néant dit le graveur dans la planche 69: Nada. Ello dirá ${ }^{35}$ où un cadavre entouré d'une masse confuse d'ombres menaçantes tient dans ses mains un papier sur lequel se lit un seul mot: « Rien ». Frédéric, après s'être perdu dans des champs de boue, des maisons vides et des chemins qui ne mènent nulle part, ne trouvera que le néant au terme de son itinéraire vital. Le bourbier sans gloire qui y met fin n'est pas sans évoquer à la fois l'épigraphe de Céline ${ }^{36}$, et l'approche désabusée et grinçante qui est celle du Voyage au bout de la nuit: "Avec amertume, Frédéric considéra qu'il avait été long ce chemin pour finir écrasé dans la boue comme un chien ${ }^{37}$.

18 La condamnation de la guerre unit les deux créateurs par-delà les siècles écoulés. Pour mieux marquer sa filiation avec le peintre, Pérez-Reverte choisit la même échelle chromatique que Goya, son récit se décline en gris et noir : la nuit, une aube sale, une journée pluvieuse, les fumées d'incendie, l'obscurité du bois et de nouveau la nuit constituent le décor de ce drame en un acte. Même les couleurs des uniformes sont à peine mentionnées, tout n'est qu'une symphonie en gris : «des champs d'oliviers couleur de cendre " «le ciel couleur de plomb $»^{38}$, «La terre était grise, la fumée grise et le ciel gris $\aleph^{39}$. Ces didascalies reviennent de façon lancinante ${ }^{40}$ et rappellent aussi l'atmosphère humide de la bataille de Waterloo chez Stendhal et le terrain boueux des opérations désordonnées décrites par Céline dans Le Voyage au bout de la nuit. Seuls un ciel de plomb et le brouillard qui rend toute visibilité impossible semblent convenir à l'univers de mort qu'est un champ de bataille. On peut d'ailleurs remarquer que tous les récits de guerre de Pérez-Reverte ( $\mathrm{El}$ sol de Breda et Territorio Comanche) reprennent la même symbolique et ont pour cadre un horizon fermé comme cette voûte de pierres si présente dans les gravures goyesques. «[U]ne voûte grise ${ }^{41}$ qui semble écraser les créatures et que Pérez-Reverte évoque lui aussi à maintes reprises.

Quelques exemples illustrent tout particulièrement les rapprochements qui peuvent être établis entre les gravures de Goya et le texte revertien. Ainsi, l'image récurrente tout au long de la narration, obsédante, cauchemardesque, du cadavre de Juniac semble immédiatement importée des Desastres de la guerra de Goya :

Juniac, tel était le nom de l'infortuné, était entièrement nu, attaché par les pieds à un arbre, la tête pendant à quelques pouces du sol. On lui avait ouvert le ventre, et les intestins grouillants de mouches s'étaient répandus, atrocement déchiquetés. ${ }^{42}$

La description qui suit fonctionne comme métatexte des estampes goyesques et fait un macabre pendant aux gravures: Fuerte cosa es, Que hai que hacer más, Esto es peor et Grande hazaña! con muertos ${ }^{143}$ où des corps empalés et mutilés sont exposés à titre d'exemple :

Les scènes de rebelles pendus par les patrouilles d'avant-garde, témoins muets, aveugles et immobiles, la langue pendante et les yeux exorbités, les corps nus, noirs, assiégés de mouches étaient devenues fréquentes au passage des troupes de l'Empereur.

Dans cette autre hypotypose d'un réalisme saisissant, selon une technique proche $\mathrm{du}$ travelling cinématographique, Pérez-Reverte se livre à un commentaire halluciné de l'œuvre du peintre comme le révèle aussi la disposition graphique du texte. Au rythme de la construction impersonnelle «il y avait», défilent les images des Desastres de la guerra:

\section{La guerre}


Il y avait deux Espagnols pendus aux branches les plus hautes d'une oliveraie ${ }^{44}$. Il y avait au loin des fermes qui brûlaient au loin ${ }^{45}$, des chevaux morts ${ }^{46}$, des uniformes verts, bruns, et bleus éparpillés partout ${ }^{47}$. Il y avait un canon renversé, la bouche enfoncée dans la boue, encloué, rendu inutilisable par l'ennemi, sans doute, avant d'être abandonné. Il y avait un soldat français, étendu sur le dos au bord du chemin, les yeux grands ouverts, les cheveux ruisselants et les mains crispées, dont les entrailles, atteintes par un éclat de mitraille, se répandaient sur ses cuisses inertes. Il y avait un blessét8 assis sur une pierre, une capote sur les épaules et le regard absent qui refusait l'aide d'un camarade debout près de lui qui semblait vouloir le convaincre de poursuivre sa route jusqu'à l'hôpital. ${ }^{49}$

Les descriptions, mais aussi certaines des images barbares qui se sont gravées à tout jamais dans la mémoire de Frédéric, semblent surgir du monde pictural de Goya. Ainsi le prêtre sacrifié en représailles après que quelqu'un a abattu la jument de Létac lors de la traversée d'un hameau convoque le souvenir des gravures 34 et 38 : Por una navaja et Bárbaros $^{50}$. On pourrait multiplier les exemples de mises en regard des citations textuelles et iconographiques car tout le texte revertien est tissé à partir de relations intersémiotiques de renvoi et d'échange avec Los desastres de la guerra facilement repérables par un récepteur doté des compétences requises. Le dénouement du roman lui-même trouve son écho dans la planche 28, intitulée "Populacho", "Plèbe ", représentant un groupe d'hommes et de femmes armés de fourches et de gourdins qui s'acharnent sur un corps à terre :

Et quand il vit approcher de la lisière du bois le groupe de paysans armés de faucilles, de bâtons et de couteaux, il se leva lentement, en redressant la tête, il regarda leurs visages olivâtres et attendit, immobile et serein. Il pensait au grandpère Glüntz, au hussard blessé sous le grand chêne. Et il n'éprouvait plus qu'une indifférence fatiguée. ${ }^{51}$

Chez Goya et chez Reverte, l'atmosphère rendue provoque une profonde répulsion chez le récepteur. Même si le dessin de Goya reste sobre, le spectateur est confronté à une réitération thématique qui épuise sa capacité à supporter la vision de la souffrance. Gravure après gravure, la charge émotionnelle se fait intolérable. Chez l'écrivain de Carthagène, on assiste au même processus d'accumulation auquel s'ajoute une poétique de l'exagération dont l'objectif est de traduire l'anéantissement qui s'est déroulé sous ses yeux pendant treize ans. La même interprétation apocalyptique des événements est au cœur de la création de l'artiste et du romancier. Ce que décrit Reverte est un véritable voyage au bout de la nuit, au bout de l'enfer ${ }^{52}$. Et l'iconographie sous jacente dote le texte revertien d'une expressivité et d'une émotivité qui contribuent à créer un effet de réel.

Dans $E l$ húsar, Histoire et fiction s'entrelacent pour produire une narration au sens éminemment historique. Le passé diégétisé, une instantanée sur la guerre de l'Indépendance, passé ressuscité à partir de l'imaginaire iconographique d'un peintre contemporain des événements relatés, acquiert non seulement une vie propre mais entretient un lien étroit avec les créatures fictionnelles qui le peuplent, et établit une relation sentie avec le présent. Car, à l'instar de Benito Pérez Galdós, Pérez-Reverte est persuadé que la société d'aujourd'hui plonge ses racines dans le passé et que la connaissance de ce passé lui permettra d'affronter avec plus de lucidité et de responsabilité le futur comme le confirment ces propos de l'auteur :

J'ai la conviction profonde qu'on ne peut rien comprendre de notre présent et de notre futur si on ne connaît pas notre passé, si nous ne mettons pas notre vision 
présente dans une essentielle perspective historique. L'Histoire est comme une puissante lumière éclairant notre présent. ${ }^{53}$

L'auteur de El Húsar interprète donc le roman historique comme « préhistoire concrète du présent ». En effet, dans ce roman, publié dans une Espagne en proie au « desencanto , au desenchantement postmoderne, il tente de redonner au lecteur espagnol des repères identitaires en l'invitant à une réflexion sur ce qui fait son idiosyncrasie, car cet épisode de la Guerre d'Indépendance constitue pour l'écrivain un des moments privilégiés où un sursaut d'orgueil national secoue le pays. Ainsi, le présent du lecteur donne sa signifiance au texte. En réactualisant un moment-clé de l'histoire d'Espagne, le discours historique de notre romancier se propose la récupération d'une mémoire fondatrice, induit une quête identitaire, et se donne pour but de fédérer une Espagne désenchantée. Cette réactualisation se fait à partir de questionnements qui sont ceux du présent du scripteur. Sans nier les particularismes, Pérez-Reverte veut redonner à l'Espagnol le sentiment d'appartenir à une communauté nationale: "Ce qui lie les Espagnols c'est leur histoire" répète-t-il avec insistance depuis sa tribune journalistique de El Semanal. Il invite donc son récepteur à retrouver ses racines en se tournant vers un moment héroique de son passé.

Dans sa pratique du roman historique, Pérez-Reverte se donne donc quelques objectifs majeurs. Témoigner d'un temps révolu parce qu'il est essentiel pour la construction d'une identité personnelle et nationale. Rendre hommage à ceux qui ont précédé parce qu'ignorer leurs souffrances revient à nier leur existence. Retrouver dans un passé commun des liens fédérateurs. Reconstruire l'Histoire parce que c'est sans doute le seul point d'ancrage, la seule bouée salvatrice dont dispose l'homme de l'époque postmoderne pour tenter de comprendre un présent dégradé.

\section{BIBLIOGRAPHIE}

ARISTOTE, Poétique, Paris, Gallimard, 1996.

CELINE Louis-Ferdinand, Voyage au bout de la nuit, Paris, Gallimard, 1952.

PAVEL, Thomas : Univers de la fiction, Paris, Seuil, 1988.

PÉREZ-GALDÓs, Benito, Bailén, in La guerra de la Independencia, Barcelona, Brughera, 1981, p. 115-171.

PÉREZ- REVERTE, Arturo:

El húsar, Madrid, Akal, 1986.

El húsar in Obra breve/1, Madrid, Alfaguara, 1995, p. 21-188.

Le Hussard, traduit de l'espagnol par François Maspero, Paris, Seuil, 2005.

ROUX Georges, Napoléon et le guêpier espagnol, Paris, Flammarion

Site de l'Université de Zaragoza sur l'œuvre de Goya.

http://goya.unizar.es/InfoGoya/Obra/Desastres.html 


\section{NOTES}

1. El húsar, Madrid, Akal, 1986, 173 p. Édition utilisée : El húsar in Obra breve/1, Madrid, Alfaguara, 1995, p. 21-188.

2. Las Aventuras del capitán Alatriste Série composée de : El capitán Alatriste, Madrid, Alfaguara, 1996, 237 p. Limpieza de sangre, Madrid, Alfaguara, 1997, 251 p. El sol de Breda, Madrid, Alfaguara, 1998, 254 p. El oro del rey, Madrid, Alfaguara, 2000, 267 p. El caballero del jubón amarillo, Madrid, Alfaguara, 2003, 348 p. Corsarios de Levante, Madrid, Alfaguara, 2006, $348 \mathrm{p}$.

3. La carta esférica, Madrid, Alfaguara, 2000, 590 p. La reina del sur, Madrid, Alfaguara, $2002,542 \mathrm{p}$.

4. El maestro de esgrima, Madrid, Mondadori, 1988. El maestro de esgrima, Madrid, Alfaguara, 1992, 275 p. La tabla de Flandes, Madrid, Alfaguara, 1990, 416 p. El club Dumas, Madrid, Alfaguara, 1992, 493 p. La piel del Tambor, Madrid, Alfaguara, 1997, 589 p.

5. Cabo Trafalgar, Madrid, Alfaguara, 2004, $260 \mathrm{p}$.

6. Un día de cólera, Madrid, Alfaguara, 2007, $401 \mathrm{p}$.

7. Arturo Pérez-Reverte, Le Hussard, traduit de l'espagnol par François Maspero, Paris, Seuil, 2005, $193 \mathrm{p}$.

8. Cette note qui figure dans la première édition a été supprimée dans les suivantes ainsi que dans la traduction française de François Maspero auquel nous empruntons toutes les traductions.

9. «Es posible que los especialistas puntillosos descubran ciertas inexactitudes en la historia que acabo de referir. No sería extraño puesto que en 1808 no se libró en Andalucía ninguna batalla con las características de la descrita en esta novela, si exceptuamos el encuentro de Bailén, que tuvo lugar en circunstancias muy diferentes. La denominación de las unidades francesas que intervienen en la narración ha sido por otra parte, adjudicada al azar, a causa de razones evidentes en una trama de ficción como la que nos ocupa. Hay, sin embargo una excepción: el $4^{2}$ Regimiento de Húsares estuvo efectivamente destacado en España entre 1808 y 1813, operando en acciones como Belchite, Alcañiz, Estela, Chiclana, Sagunto, Yecla y Paso de Ordás. Ignoro si en algunos de esos combates resultó aniquilado su Primer Escuadrón, aunque tengo serios motivos para dudarlo, ya que no he hallado constancia de ello en ninguno de los numerosos documentos consultados...Sin embargo, la ficción confiere a veces al autor el divertido privilegio de hacerle trampas a la Historia." El húsar, Madrid, Akal, $7^{\mathrm{a}}$ edición 2002, p. 171.

10. Nous renvoyons à l'Épisode National de Benito Pérez-Galdós dans lequel le narrateur Gabriel Araceli, jeune soldat des troupes espagnoles relate la bataille de Bailén : Bailén, in La guerra de la Independencia, Barcelona, Brughera, 1981, p. 115-171.

11. Aristote, Poétique, Paris, Gallimard, 1996, p.93

12. Voir : Thomas Pavel, Univers de la fiction, Paris, Seuil, 1988, p. 59-93.

13. Le Hussard, traduction de F. Maspero, op. cit., p. 115. "Era lamentable, sin embargo, que napoleón no hubiese comprendido todavía que a España no se la podía medir con el mismo patrón que al resto de los países europeos. [...] España es una nación muy vieja, orgullosa y leal a sus mitos, estén justificados o no. Bonaparte está tan acostumbrado a ver arrodillarse a los pueblos, que no puede concebir, y ése es el error de apreciación, que al sur de los Pirineos hay una raza dispuesta a no aceptar su voluntad." El húsar, op. cit., p. 119- 120.

14. Georges Roux, Napoléon et le guêpier espagnol, Paris, Flammarion, 1970, p.78. 
15. «[...] en este país hasta los perros, las aves, el sol y las piedras son nuestros enemigos. » A. Pérez-Reverte, El húsar, op. cit., p. 41-42.

16. Le Hussard, op. cit., 33. "Sin embargo, todos estaban convencidos, Frederic entre ellos, de que tal situación no se prolongaría durante mucho tiempo. La dureza y la profusión de castigos ejemplares no tardarían en hacer volver las aguas a su cauce. Todo era cuestión de ahorcar más, arcabucear más a aquella canalla inculta y fanática, concluyendo de una vez la pacificación de España para seguirse dedicando a más gloriosas empresas. ». Ibid., p. 44-45.

17. Le Hussard, op. cit., p. 41. «Creo...creo que un hombre como tú, o como yo, sólo se justifica, sólo encuentra su razón de ser, cabalgando con las riendas entre los dientes, pistola en una mano y sable en la otra, aullando su grito de guerra en nombre del Emperador. » El húsar, op. cit., p. 52.

18. «Pour Frédéric Glüntz, jeune sous-lieutenant nourri de tous les vastes rêves de gloire que peut contenir une solide tête de dix-neuf ans, le colonel Letac représentait ce qu'il aurait voulu devenir tandis que Michel de Bourmont était ce qu'il aurait voulu être, l'incarnation d'un rang personnel et social que jamais il n'atteindrait, même s'il parvenait à faire fortune. " Le Hussard, op. cit., p.19.

«Para Frédéric Glüntz, joven subteniente nutrido con todos los dilatados sueños de gloria que podía albergar una sólida cabeza de diecinueve años, el coronel Letac era lo que le gustaría llegar a ser. Michel de Bourmont era justamente aquello que habría querido poder ser, encarnación de un rango personal y social que jamás, aunque en el futuro curso de su vida lograse hacer fortuna, alcanzaría. » El húsar, op. cit., p. 32.

19. Lukács Georges, Le roman historique, op. cit., p. 44.

20. «En la carrera de las armás buscaba la cristalización de un anhelo superior, de un sentimiento que lo arrancaba de las comodidades de la vida burguesa y lo ponía en el camino de la heroïcidad, de los sentimientos nobles, del sacrificio supremo. Había entrado en la milicia como quien entra en religión, abrazando su sable como quien abraza una cruz. Y si los sacerdotes o los pastores aspiraban a ganar el cielo, él aspiraba a ganar la gloria: la admiración de sus camaradas, el respeto de sus jefes, la propia estimación a través de experimentar ese bello y desinteresado sentimiento de vivir con la conciencia de que era dulce y hermoso pelear y sufrir y quizá morir por una idea. La Idea. » El húsar, op. cit., 112.

21. Le Hussard, p. 192. «Los viejos soldados, eso lo descubría ahora siempre tenían razón. Por eso se callaban. Ellos sabían, al diablo con todo. Pero no se lo contaban a nadie; eran viejos zorros astutos. Que cada uno aguantara su vela, que cada cual aprendiera por sí solo. En ellos no había valor; había indiferencia. Estaban al otro lado del muro, más allá del bien y del mal, como el abuelo de Frédéric, el viejo Glüntz que se dejó morir cansado de esperar la muerte. No había nada más que hacer, el camino estaba espantosamente claro. Honor, Gloria, Patria, Amor...Había un punto sin retorno, al que se llegaba tarde o temprano, en el que todo se tornaba superfluo, adquiría sus límites precisos, su exacta dimensión. » El húsar, p. 187.

22. Le Hussard, p. 41. « Me preocupa haber llegado hasta aquí para caer de forma oscura y sin gloria, asesinado en un camino solitario por chusma campesina como el pobre Juniac, en vez de hacerlo cabalgando tras el águila del Regimiento, a cielo abierto y rodeado por los camaradas, de un limpio sablazo o de un tiro en el pecho, de pie, con las espuelas en su sitio, el arma en la mano y la boca llena de sangre, como mueren los hombres. » El húsar, p. 53. 
23. Le Hussard, p.33. «[...] brillantes cargas de caballería, movimiento de grandes unidades, batallas con nombres gloriosos que figurarían en los libros de Historia y que supondrían para Frederic Glüntz los peldaños del honor y de la fama. » El húsar, p. 45.

24. Ibid., p. 23.

25. Les Désastres de la guerre, titre de la série de gravures réalisées par Goya en 1814.

26. «Yo lo vi » est le titre de la planche 44. Voir sur Internet le site : Info Goya 96 de l'université de Zaragoza. http://goya.unizar.es/InfoGoya/Obra/Desastres.html 27. pl. 38. Voir http://goya.unizar.es/InfoGoya/Obra/Desastres.html

28. « Le sommeil de la raison engendre des monstres » : Los caprichos, pl. 43, voir http:// goya.unizar.es/InfoGoya/Obra/Caprichos.html

29. «Les conséquences » Los désastres de la guerra, pl. 72 : voir http://goya.unizar.es/ InfoGoya/Obra/Desastres.html .

30. «Un monstre féroce », http://goya.unizar.es/InfoGoya/Obra/Desastres.html

31. El húsar, op. cit., p. 186.

32. «A Claude, mi viejo compañero de guerras ajenas y de caminos que no llevan a ninguna parte. », p. 21.

33. «Moi, d'abord la campagne, faut que je le dise tout de suite, j'ai jamais pu la sentir, je l'ai toujours trouvée triste avec ses bourbiers qui n'en finissent pas et ses chemins qui ne mènent nulle part. Mais quand on y ajoute la guerre en plus, c'est à pas y tenir. ", Louis-Ferdinand Céline, Voyage au bout de la nuit, Paris, Gallimard, 1952, p.13.

34. "Tristes pressentiments de ce qui va arriver. " pl.1, http://goya.unizar.es/InfoGoya/ Obra/Desastres.html

35. pl. 69, http://goya.unizar.es/InfoGoya/Obra/Desastres.html

36. Voir p. 87.

37. Con amargura, Frédéric consideró que había sido un largo camino para terminar aplastado en el lodo como un perro. " Pérez-Reverte Arturo, El húsar, op. cit., p. 174.

38. « el cielo plomizo ». Ibid., p. 139 et « el cielo color de plomo » p.142.

39. « La tierra era gris, el humo gris, y el cielo gris. » Ibid., p. 144.

40. «El cielo color ceniza, veteado de negros nubarrones, gravitaba sobre la tierra como si estuviese preñado de plomo. Una fina llovizna comenzó a caer sobre los campos, velando el paisaje gris.» « le ciel couleur de cendre, veiné de gros nuages noirs, gravitait sur la terre comme s'il était chargé de plomb. Une fine bruine commença à tomber sur les champs, jetant un voile gris sur le paysage. » Ibid., p. 75.

« El cañón seguía rugiendo en la distancia, al otro lado del horizonte gris. » « Le canon rugissait encore dans le lointain, de l'autre côté d'un horizon gris », Ibid., p. 82.

41. « aquella bóveda gris », ibid., p. 147.

42. Le Hussard, op. cit., p. 32. «[...] estaba completamente desnudo, atado por los pies a un árbol con la cabeza a dos palmos del suelo. Le habían abierto el vientre con su propio sable, y los intestinos cubiertos por un enjambre de moscas, colgaban como un despojo de horror. ", El húsar, op. cit., p. 44.

43. Respectivement : pl. 31, pl. 33., pl. 37, pl.39. http://goya.unizar.es/InfoGoya/obra/ Desastres.html

44. Echo de la planche 36: Tampoco.

45. Escapan entre llamas. pl. 41.

46. Siempre sucede, pl. 8.

47. Tanto y más pl. 22, et Lo mismo en otras partes, pl. 23.

48. Curarlos y a otra, pl. 20. 
49. Le Hussard, op. cit. : « La guerra Había un olivar del que pendían dos españoles de las ramas más altas Había granjas que humeaban a lo lejos, caballos muertos, uniformes verdes, pardos y azules de cadáveres diseminados por todas partes. Había un cañón volcado, con la boca hundida en el barro, con un clavo en el orificio, inutilizado sin duda por el enemigo antes de abandonarlo. Había un soldado francés tendido boca arriba a un lado del camino, con los ojos muy abiertos, el cabello húmedo y las manos engarfiadas, cuyas entrañas, abiertas por una esquirla de metralla, se desparramaban sobre sus muslos inertes. Había un herido sentado en una piedra con un capote sobre los hombros y la mirada ausente, que negaba con la cabeza a un compañero que, de pie a su lado, parecía querer convencerlo para que prosiguiera camino hacia el hospital. (...) » El húsar, op. cit., p. 146.

50. http://goya.unizar.es/InfoGoya/Obra/Desastres.html

51. "Y cuando vio acercarse por la linde del bosque al grupo de campesinos armados con hoces, palos y navajas, se levantó despacio con la cabeza erguida, miró sus rostros cetrinos y aguardó, inmóvil y sereno. Pensaba en el abuelo Glüntz, en el húsar herido bajo la gran encina. Y no sentía más que una cansada indiferencia." El Húsar, op. cit., p. 187-188.

52. Voir El húsar, op. cit., p. 157.

53. Entretien : Arturo Pérez-Reverte: « Ma vraie patrie, c'est mon enfance », op. cit., p.103.

\section{RÉSUMÉS}

En 1986, Arturo Pérez-Reverte publie El húsar, un récit assez bref qui relate une bataille fictive qui se serait déroulée en Espagne pendant l'invasion napoléonienne. Dans ce drame, en un acte et sept tableaux, le hussard Frédéric Glüntz, archétype des jeunes recrues de l'armée impériale fait le cruel et mortel apprentissage de la guerre.

$\mathrm{Si}$, comme le revendique l'auteur, la bataille est purement fictionnelle, la Guerre de l'Indépendance est indéniablement attestée par l'Histoire. Entre mensonge et vérité, le romancier élabore donc un texte en continuelle tension entre historicisation de la fiction et fictionalisation de l'Histoire dans lequel le contexte historique est investi d'une fonction diégétique et engendre des créatures poétiques, « héros moyens » conscients de leur inscription dans l'Histoire.

$\mathrm{Au}$ fil du roman, le passé diégétisé, soigneusement reconstitué grâce à une documentation rigoureuse, puisée à des sources historiographiques et iconographiques comme l'œuvre gravé de Goya, s'impose comme le témoignage vécu d'une journée de la guerre de l'Indépendance, tragique et fatale.

En 1986, Arturo Pérez-Reverte publica El húsar, un relato de corta extensión que narra una batalla ficticia que supuestamente habría tenido lugar en España durante la invasión napoleónica. En este drama en un acto y siete cuadros, el húsar Frederic Glüntz, arquetipo de los jóvenes reclutas del ejército imperial hace el cruel y mortal aprendizaje de la guerra.

$\mathrm{Si}$, como bien lo reivindica el autor, la batalla es totalmente ficcional, innegablemente La Historia atestigüa la existencia de la Guerra de la Independencia. Entre mentira y verdad, el novelista elabora pues un texto en continua tensión entre "ficcionalización" de la Historia e 
"historicización" de la ficción, un texto en que el contexto histórico desarrolla una función diégética y engendra criaturas poéticas, "héroes medios" conscientes de pertenecer a la Historia. A lo largo de la novela, el pasado narrado, reconstituido con esmero y sumo rigor mediante una documentación procedente de fuentes historiográficas e iconográficas como la obra grabada de Goya, cobra valor de testimonio, de vivencia trágica y fatal de un día de la Guerra de la Independencia.

\section{INDEX}

Mots-clés : Pérez-Reverte, fiction historique, Hussard, guerre de l'Indépendance, univers fictionnel, les désastres de la guerre de Goya, Bailén Index chronologique : XXe siècle

\section{AUTEUR}

\section{MARIE-THÉRÈSE GARCIA}

Université du Sud Toulon-Var, Laboratoire Babel 